\title{
GOBERNANZA UNIVERSITARIA O COGOBIERNO: EL CASO DE LA UNIVERSIDAD DE CONCEPCIÓN DE CHILE
}

\author{
MARIOL VIRGILI LILLO* \\ FRANCISCO GANGA CONTRERAS ${ }^{* *}$ \\ KATHERINE FIGUEROA AILLAÑIR ${ }^{* *}$
}

\begin{abstract}
RESUMEN
Este trabajo se centra en la participación en la Universidad de Concepción, abordada desde el estudiante-actor, que implica desarrollar y asumir una postura activa en el pregrado donde éste forme parte de la comunidad universitaria y desde la Universidad, como institución-actor, encargado no solo de asuntos direccionales sino también de disponer y asegurar espacios de participación para que éstos «hagan universidad». Se caracteriza la «participación estudiantil», establece si éstos entienden la participación como eminentemente política y de gobierno, excluyendo otras dimensiones de participación, y concluir respecto del enfoque que la dimensión política, como primordial, más próxima a gobernanza o a cogobierno.
\end{abstract}

\footnotetext{
PALABRAS CLAVE: PARTICIPACIÓN, PARTICIPACIÓN ESTUDIANTIL, GOBERNANZA UNIVERSITARIA, COGOBIERNO UNIVERSITARIO
}

* Magíster en Administración Educacional, Magíster en Política y Gobierno (C), Subdirectora de docencia, Universidad de Concepción.

E-Mail: mvirgili@udec.cl.

** Administrador Público, Licenciado en Administración, Magíster en Administración de Empresas, DEA en Organización de Empresas, Doctor en Administración de Empresas y Postdoctorado en Ciencias Humanas. Actualmente es académico jornada completa del Departamento Ciencias del Desarrollo de la Universidad de Los Lagos, Campus Santiago.

E-Mail: fganga@ulagos.cl.

*** Administrador Público, Magíster en Política y Gobierno @, Departamento de Administración Pública y Ciencia Política, Facultad de Ciencias Jurídicas y Sociales, Universidad de Concepción.

E-Mail: katfigueroa@udec.cl. 


\title{
GOVERNANÇA UNIVERSITÁRIA OU COGOVERNO O CASO DA UNIVERSIDAD DE CONCEPCIÓN
}

\begin{abstract}
RESUMO
Este trabalho está centrado na participação na Universidad de Concepción a partir de duas perspectivas, a primeira é da perspectiva do estudante-ator, que implica desenvolver e assumir uma postura ativa na graduação onde este seja parte da comunidade universitária. A outra é a partir da perspectiva da universidade como instituição-ator, encarregada não só da direção senão que também de dispor e garantir espaços de participação para que os estudantes «façam universidade». Caracteriza-se a «participação estudantil», estabelece se os estudantes entendem a participação como eminentemente política e de governo, excluindo outras dimensões de participação, e conclui a respeito do enfoque que a dimensão política tem, se mais próximo da governança ou do cogoverno.
\end{abstract}

\author{
PALAVRAS CHAVE: PARTICIPAÇÃO, PARTICIPAÇÃO ESTUDANTIL, \\ GOVERNANÇA UNIVERSITÁRIA, COGOVERNO UNIVERSITÁRIO
}

\section{UNIVERSITY GOVERNANCE OR COGOVERNMENT THE CASE OF UNIVERSIDAD DE CONCEPCIÓN}

\begin{abstract}
This work is focused on participation in Universidad de Concepción from two main stances; on the one hand, from a student-actor standpoint, involving the development and assumption of an active stance on the undergraduate program in the university community, as part of it. On the other hand, from the university, as an institution-actor standpoint, not only in charge of management but also of arranging and ensuring participation opportunities for students in order to be an «active part of university». The concept «student participation» is characterized, also the idea if students understand the participation as eminently political and governmental, excluding other participative dimensions is established, and the approach of the political dimension, closer to governance or cogovernment is concluded.
\end{abstract}

KEY WORDS: PARTICIPATION, STUDENT PARTICIPATION, UNIVERSITY GOVERNANCE, UNIVERSITY COGOVERNMENT 


\section{INTRODUCCIÓN}

ACTUALMENTE, PARA LA CONSTRUCCIÓN de una sociedad democrática resulta esencial la existencia de ciudadanos activos, comprometidos con la comunidad, cívicamente responsables y conscientes de su rol en la construcción de la sociedad, razón por la cual muchas investigaciones centran sus objetos de estudio en el proceso educativo y formativo de niños y jóvenes de nuestra sociedad, pues se ha demostrado que la experiencia que éstos tengan en los distintos niveles educativos en materias relacionadas con la democracia y ciudadanía, es definitoria en la cultura política que estos adquieran, en la importancia que ellos otorgarán a la democracia y a la vinculación con la sociedad y el hacer común. Por ello, considerando las características estructurales y organizativas de las instituciones universitarias, es que el espacio universitario como contexto ofrece «una oportunidad idónea para la práctica de la democracia a pequeña escala» (Martín, 2007).

La participación está directamente relacionada con la democracia, ello permite otorgar suficiente importancia a su análisis en el ámbito universitario, considerando que en el sistema democrático chileno, un fenómeno conocido es el escaso interés por participar de los jóvenes chilenos. El desencanto de la democracia actual es evidente y aparentemente transversal en ellos. Sin embargo, esto no ha implicado una disipación de los estudiantes universitarios en la vida social, sino más bien, se ha acentuado la participación a través de vías alternativas, también expresiones democráticas, como las movilizaciones, planteamiento de demandas y trabajo conjunto con otros sectores de la sociedad, de tal modo de empoderar a los estudiantes universitarios como un actor determinante en la sociedad.

Crecientes han sido las manifestaciones de los estudiantes universitarios en cuanto a plantear sus demandas educativas, sociales y políticas, entre otras, a nivel país. Sin embargo, a escala universitaria también se han planteado reivindicaciones, a la luz de los mismos conflictos, se ha puesto en la palestra un enérgico reclamo estudiantil cuyo foco se ha centrado en redefinir y fortalecer el «rol del estudiante en la universidad», ampliando los márgenes de participación, exigiendo un mayor empoderamiento que les permita ser actores determinantes en la toma de decisiones nivel de gobierno universitario, acrecentando los ámbitos de participación que hasta hoy contemplan los reglamentos y estructuras institucionales de la mayor parte de las universidades chilenas. 
En este contexto, emerge una interrogante acerca de cuál es el contenido de esta nueva demanda, y principalmente acerca de lo que estos entienden por «participación estudiantil», pues aparentemente no existe claridad respecto de su contenido muldimensional que parece estar en franco abandono, disyuntiva que constituye el objeto principal de esta investigación, que se advierte como un primer esfuerzo en orden a caracterizar lo que los estudiantes de la Universidad de Concepción (UDEC) entienden por «participación estudiantil», su contenido e incidencia en el gobierno universitario así como sus limitantes y nuevas perspectivas.

El presente trabajo, desde la noción de gobernanza universitaria, explora las opiniones de los estudiantes buscando resultados que no solo permitan clarificar el concepto, sino que también delimitar su aplicación. Como estudio exploratorio, pretende iniciar el proceso para avanzar en el conocimiento de este tema y facilitar estudios venideros, como también determinar la importancia de la participación estudiantil universitaria en un escenario actual de crecientes desafecciones e inseguridades, donde emerge la necesidad de fortalecer la sociedad civil y el interés por dotar de contenido y valores colectivos al concepto de ciudadanía, que también comprende el de ciudadanía universitaria.

\section{DESARRollo}

\section{a) La participación estudiantil, marco referencial y conceptual}

i) Democracia y participación estudiantil

El desarrollo y perfeccionamiento de la democracia se ha constituido como el principal objetivo en Chile aún antes de 1990, se ha presentado como la piedra angular para el desarrollo de las sociedades, inherente a su naturaleza, empero su incorporación y ejercicio no ha estado exento de complejidades y discrepancias. Ha sido definida como «el conjunto de reglas cuya observancia es necesaria con el objeto de que el poder político sea distribuido efectivamente entre la mayor parte de los ciudadanos y por otra, el ideal en el cual un gobierno democrático debería inspirarse, que es el de igualdad» (Bobbio, 2000).

La democracia se ha construido como una garantía en la vida de quienes forman parte de la sociedad, así, para Robert Dahl (1999) «la democracia se presenta como el sistema político más aceptable debido a que garantiza, al menos en diez puntos, ventajas por sobre otros 
sistemas políticos de gobierno, estos son: a) Evita la tiranía; b) Garantiza derechos esenciales; c) Otorga libertad general; d) Promueve la autodeterminación; e) Proporciona autonomía moral; f) Incentiva el desarrollo humano; g) Protege los intereses personales esenciales; h) Fomenta altos grados de igualdad política; i) Produce la búsqueda de la paz; j) Genera prosperidad en las sociedades».

La gobernabilidad democrática, tiene como pilar fundamental la participación, sin participación no existe democracia, por ello, ha tomado un papel determinante en todos los ámbitos de la sociedad, incluyendo el universitario, como instancia de formación y aprendizaje, pues parece existir consenso entre los académicos y agentes formuladores de políticas públicas educacionales, respecto de la necesidad de desarrollar las capacidades y pensamiento crítico de los estudiantes, enfoque que permitirá la formación de una cultura cívica democrática, con ciudadanos activos y conscientes, conocedores de sus derechos, deberes y libertades en la sociedad.

Desde un punto de vista empírico, diversos estudios demuestran que la universidad es uno de los espacios de socialización secundaria en los que el estudiante puede desarrollar actitudes, aprender habilidades y vivir experiencias útiles para su participación como ciudadano en una democracia (Andolina et al., 2007; Astin, Sax y Avalos, 1999), por lo que la forma en que los estudiantes «hacen universidad»; es decir, la forma en que participan de la etapa universitaria, determinará en gran modo el futuro de nuestra democracia.

Diversos han sido los esfuerzos por indagar respecto de la participación estudiantil, su contenido y mecanismos en otros países, en Europa, la Declaración de Praga (2001), documento en el que se empiezan a gestar las líneas maestras del proceso de Bolonia, pone énfasis en el llamado realizado por los Ministros y Ministras a la participación del alumnado en el proceso de construcción del Espacio Europeo de Educación Superior (EEES) y las universidades, y la necesidad de influir en su organización y contenido. A su vez, resulta de interés también la mención de la Declaración de Berlín (2003) donde no solo se recoge la participación del estudiantado en la gobernanza de la Universidad, sino el acuerdo de incluir al alumnado en los Sistemas de Garantía de Calidad Nacionales (mencionado de nuevo en la Declaración de Bergen del 2005). En 2011, el Ministerio de Educación impulsó la implantación de un sistema de atención integral al alumnado y a los jóvenes titulados universitarios en el marco del EEES, los programas de actuación para la participación del alumnado en la vida universitaria tanto a nivel político, 
asociativo, como en actividades académicas, culturales, sociales, de voluntariado, etcétera, así como la programación, gestión y evaluación del programa de apoyo a las actividades de las organizaciones estudiantiles (Universidad del País Vasco, 2012).

\section{ii) La situación de Latinoamérica y Chile}

En Latinoamérica, la llegada de las universidades sigue las líneas experimentales europeas, a partir de 1518 comienza literalmente a trasplantarse e incorporarse en diversos países, el modelo educacional español, como una manifestación de la consolidación de la conquista, sin embargo la forma de funcionamiento y desarrollo fue disímil entre la mayoría de los países. En una primera etapa, el acceso a la universidad correspondía únicamente a la elite, consecuencia de ello, existían pocos alumnos y con ello las decisiones eran tomadas exclusivamente por los profesores (Brunner, 1990). Poco a poco se aumentó el número de universidades y con ello la cantidad de estudiantes universitarios, en 1918 se advierte el primer quiebre en la forma de gobierno universitario, con el histórico movimiento estudiantil de la Universidad Nacional de Córdoba en que los estudiantes exigen el término del autoritarismo y logran aumentar las libertades y participación de los estudiantes en la toma de decisiones a través de la implementación del cogobierno universitario, marcando un hito en las bases de la participación estudiantil universitaria. Casi 50 años más tarde, se advierten en Chile, demandas similares impulsadas por el movimiento estudiantil de la Universidad Católica de Chile y Valparaíso.

En Chile, los primeros esfuerzos por la incorporación, en ciertos casos, y la extensión del ámbito de la participación estudiantil, en otros, se remontan al año 1918, sin embargo esos primeros esfuerzos no rinden frutos sino hasta 1967 en que tiene lugar el movimiento estudiantil y de reforma universitaria, a cuya virtud, en el año 1968 se logra en todas las universidades del país, la elección democrática del rector y demás autoridades universitarias, como el consejo superior integrado por los decanos, a través de un claustro pleno, que considera la participación de docentes, no docentes y estudiantes, haciendo partícipes a los estudiantes de la toma de decisiones que se enfocaba en aquellos años a la conexión y compromiso de la universidad con la comunidad y a la modernidad de estas instituciones. Sin embargo, este ámbito de participación estudiantil se suspende y sustituye por un mecanismo de designación de rectores y autoridades por el gobierno 
central, incorporado después del 11 de septiembre de 1973, por la dictadura de Augusto Pinochet. En este contexto, la movilización estudiantil se ha constituido como la principal forma, con que cuentan los estudiantes en la exigencia de una mayor participación estudiantil, para fracturar el marco institucional y derrocar las estructuras anacrónicas del funcionamiento de las universidades chilenas.

Después de 1973, las universidades públicas sufrieron un proceso de fragmentación, que se agudizó en 1981 en que se dicta una nueva legislación universitaria en Chile que tiene como finalidad el crecimiento del sistema de educación superior a través de: la admisibilidad e incorporación al sistema educativo de las universidades privadas sin fines de lucro, el autofinanciamiento universitario, dando inicio al aporte fiscal indirecto, eliminación de los espacios de actividad política; y la negación de la participación de estudiantes y funcionarios en las elecciones de autoridades y consejos superiores.

En el período de 1990 a 1995, el movimiento estudiantil constituido hasta entonces como un poderoso mecanismo de participación y presión en la consecución de las demandas estudiantiles, manifestó una decadencia, pues se había logrado la democracia que se presentaba como la única bandera de lucha de los estudiantes universitarios. Los bajos niveles de participación en las universidades provocan incluso el debilitamiento de la Confederación de Estudiantes de Chile (CONFECH), la cual solo retomaría fuerzas en 1997 para enfrentar las intenciones de privatizar la educación pública, luego en 1999 para enfrentar la saturación del fondo solidario. Sin embargo, no sería sino hasta 2003 que las universidades chilenas se preocuparían, de manera representativa, de la participación estudiantil a nivel nacional, tomando fuerza a partir de 2005 hasta la fecha, la transformación de los estudiantes universitarios en actores políticos, centrando sus esfuerzos en la elaboración de una propuesta de reforma de la educación superior y en las demandas internas, en sus casas de estudio, por ganar espacios de participación, sobre todo, en el ámbito político (CONFECH, 2012).

Como se ha señalado, en la actualidad la educación superior se encuentra regulada por estatutos orgánicos dictados en época de dictadura militar, constituyéndose como la base de una fortalecida estructura universitaria dirigida desde 1973 hasta 1990 por rectores designados, excluyendo del ámbito decisional a los estudiantes y demás estamentos universitarios. 
Manifestación jurídica de lo anterior fue la Ley 18.962 conocida como Ley Orgánica Constitucional de Enseñanza, o LOCE, ${ }^{1}$ que contempla en su artículo 49 -actualmente consagrado en términos similares en el DFL $\mathrm{N}^{\circ} 1$ de $2005^{2}$ en su artículo 56- una disposición referente a la participación política en las universidades, señalando que «la forma de gobierno de la nueva entidad deberá excluir la participación con derecho a voto de alumnos y de funcionarios administrativos, tanto en los órganos encargados de la gestión y dirección de ella, como en la elección de autoridades unipersonales o colegiadas». ${ }^{3}$

La Ley General de Educación N²0.370 dictada en el año 2009, derogó en su mayor parte el texto de la LOCE excepto en su Título III, con exclusión del párrafo 2 , y su Título IV normas referidas básicamente a la educación superior, reafirmando el contenido de la norma ya expresada. Esta ley se refiere a la participación estudiantil, declarándola como un principio del Sistema Educativo Chileno al señalar en su artículo 3 letra g): «Participación. Los miembros de la comunidad educativa tienen derecho a ser informados y a participar en el proceso educativo en conformidad a la normativa vigente». Con todo, el derecho de información y participación a que se hace referencia se encuentra limitado únicamente al proceso educativo, cultural, deportivo y recreativo, refiriéndose únicamente al derecho de asociación de los estudiantes en el ámbito político.

1 La LOCE fue dictada por Junta de Gobierno, que en aquellos años ejercía las función legislativa en Chile, y promulgada por Augusto Pinochet Ugarte, el 7 de marzo de 1990, siendo publicada en el Diario Oficial el 10 de marzo del mismo, último día de la dictadura militar.

2 Fija texto refundido, coordinado y sistematizado de la Ley $\mathrm{N}^{\circ} 18.962$, Orgánica Constitucional de Enseñanza.

3 Art. 56. Los estatutos de las universidades deberán contemplar en todo caso, lo siguiente: a) Individualización de sus organizadores; b) Indicación precisa del nombre y domicilio de la entidad; c) Fines que se propone; d) Medios económicos y financieros de que dispone para su realización. Esto último deberá acreditarse ante el Consejo Nacional de Educación; e) Disposiciones que establezcan la estructura de la entidad, quiénes la integrarán, sus atribuciones y duración de los respectivos cargos. La forma de gobierno de la nueva entidad deberá excluir la participación con derecho a voto de los alumnos y de los funcionarios administrativos, tanto en los órganos encargados de la gestión y dirección de ella, como en la elección de las autoridades unipersonales o colegiadas; f) Los títulos profesionales y grados académicos de licenciado que otorgará inicialmente, y g) Disposiciones relativas a modificación de estatutos y a su disolución. 
Sin embargo, en el año 2010 se dictó el DFL N ${ }^{\circ} 2,{ }^{4}$ normativa que en sus artículos 56 letra e), artículo 67 letra e), y artículo 75 letra e), establecen prohibiciones explícitas para la libre asociación universitaria, además de reiterar la manifestación de que tanto estudiantes como trabajadores deberán ser excluidos de la elección de autoridades así como no estarán facultados para optar a dichos cargos.

Establecido el contexto, marco jurídico y avances de la admisibilidad de la participación en el contexto universitario, resulta necesario, para efectos de esta investigación indagar respecto de los conceptos claves que constituyen las directrices de este documento y que delimitan la interpretación de los resultados que se plantearan más adelante. Los conceptos esenciales a revisar son: participación estudiantil, gobernabilidad, gobernanza, cogobierno y ciudadanía, conceptos ajustados al ámbito universitario y determinantes en la construcción de la democracia universitaria.

Cuando hablamos de participación, partimos de la base de que es un concepto multidimensional. Para delimitar su significado debemos comprender en primer lugar que se entiende por conducta participativa. Según Navarro (2012) la conducta participativa está formada por todas aquellas acciones tendientes a la asociación con otras personas en situaciones y procesos y con relación a objetivos finales claros y conscientes para ella, pero significativos para el sistema social. Se la concibe como comportamiento moral en la medida que se asume la interdependencia en el desarrollo humano y que solo en la cooperación con otros la persona adquiere un mayor ejercicio del poder, ya sea para enfrentar situaciones y problemáticas personales y colectivas o para conseguir el bienestar común y el desarrollo efectivo de las sociedades en los próximos años. La conducta participativa ha sido estudiada en distintos contextos, es importante destacar que Krauskopf (1998) en relación a la participación de los adolescentes en las políticas y programas destinados a ellos, concluyó que ésta requiere contar con sus aportes en las propuestas de iniciativas, la negociación para alcanzar objetivos trazados y la vinculación a las políticas básicas de los programas y a la articulación de los planes.

Por otra parte, la participación del estudiantado en la universidad implica asumir una postura activa donde éste quede integrado en la organización de la misma. Una de las funciones más relevantes de la

4 Fija texto refundido, coordinado y sistematizado de la Ley $\mathrm{N}^{\circ} 20.370$ con las normas no derogadas del Decreto con Fuerza de Ley $\mathrm{N}^{\mathrm{o}} 1$ de 2005. 
universidad es la función social, mediante la cual el estudiantado recibe una formación en valores de análisis, reflexión y participación democrática (Merhi, 2011).

La participación desde una concepción holística implica diversos espacios como el político, académico, cultural, deportivo, de cooperación y solidaridad con la comunidad. Desde el punto de vista político, quedan comprendida la participación electoral, como candidato en elecciones representativas o en el ejercicio del voto, en los distintos niveles eleccionarios de los órganos de representación y gobierno que ofrece la institucionalidad universitaria a nivel estudiantil, académico y directivo, así como también la participación en espacios políticos o de formación política. En el ámbito académico, incluye no sólo la realización de las actividades académicas propias del quehacer universitario, sino que además el interés por otras áreas como la investigación, ayudantías, etcétera. En el ámbito deportivo hemos considerado la práctica o seguimiento activo de algún deporte o equipo a nivel universitario. En el ámbito cultural se han incluido actividades artísticas, musicales, educativas y de entretenimiento; y finalmente, en el ámbito de voluntariado se comprenden las actividades de cooperación con la comunidad de tipo religioso, fundaciones privadas, municipales, vecinales, entre otras.

\section{iii) La gobernabilidad y la gobernanza}

La gobernabilidad o governance, se ha entendido como el ejercicio del poder o la función de gobernar. Para Tomassini (1993) se refiere a la capacidad de la autoridad para canalizar los intereses de la sociedad civil, a la interacción que se da entre ambos segmentos y por tanto a la legitimidad del primero de ellos: el gobierno. La gobernabilidad por tanto, no se limita meramente al ejercicio del poder de gobierno, si no más bien se hace extensiva a la capacidad de los gobiernos para crear las condiciones necesarias que permitan sustentar la existencia misma del gobierno, desde el punto de vista de su legitimidad, asegurando la efectividad y estabilidad de su quehacer. De hecho, las demandas en torno a la función del poder de gobierno dicen relación con la incorporación de la democracia, avanzando hacia la gobernabilidad democrática, que requiere de la incorporación de la sociedad en el ejercicio de esta función, definida por el Programa de las Naciones Unidas para el Desarrollo (2002) como «la capacidad de una sociedad de definir y establecer políticas y resolver sus conflictos de manera 
pacífica dentro de un orden jurídico vigente», reconocida como una condición necesaria del Estado de derecho, requieriéndose de instituciones basadas en los principios de equidad, libertad, participación en la toma de decisiones, rendición de cuentas, etcétera.

Por su parte, la gobernanza es un concepto multívoco, esencialmente se ha entendido como la forma en que se ejerce el poder de gobierno que determina a su vez, la toma de decisiones. Particularmente, la gobernanza a nivel de educación universitaria se entiende como «la manera en que las instituciones se hallan organizadas y son operadas internamente - desde el punto de vista de su gobierno y gestión- y sus relaciones con entidades y actores externos con vistas a asegurar los objetivos de la educación superior» (Brunner, 2011).

$\mathrm{Al}$ referirnos al cogobierno de los estudiantes en la conducción de las universidades, al que también parecen aspirar los trabajadores universitarios, no podemos dejar de referirnos nuevamente al célebre Movimiento de la Universidad Nacional de Córdoba, que en 1918 exige la existencia de la autonomia de las universidades y la gestión conjunta del gobierno universitario, reconociendo así la capacidad creadora y realizadora de sus integrantes que les permitiere autodeterminarse, traspasándose una amplia gama de facultades desde el gobierno central a cada una de las universidades respectivamente. En ese contexto, los estudiantes reformistas de 1918 plantearon el cogobierno como una forma de gobierno que integra en partes iguales a profesores y estudiantes, con la finalidad de construir la «República Universitaria» (Diodato y Martínez, 2006).

De este modo, entendemos que el cogobierno universitario demanda el gobierno de la universidad de manera compartida e igualitaria - por los distintos integrantes de la comunidad universitaria, esencialmente académicos y estudiantes - lo que denominaremos «cogobierno biestamental», aunque también es posible advertir un sector importante, que demanda la inclusión en este concepto de los trabajadores no académicos, dando lugar al «cogobierno triestamental».

Las universidades, hacen posible el análisis de la incorporación de la democracia como sistema político de gobierno en este tipo de instituciones. Es sabido que la educación fortalece la relación entre hombres y mujeres y con ello, los que éstos tienen con la sociedad, con todo, la formación de ciudadanos no se reduce únicamente a la entrega de conocimientos en algún area del saber, sino que además se refiere a la formación de valores y ética ciudadana, y más aún, con el ejercicio de éstos en el espacio universitario. 
La ciudadanía, implica el reconocimiento de principios, normas y valores que se le aseguran a un sujeto y que orientan la relación que éste tiene con la sociedad, término perfectamente admisible en la realidad universitaria y que implica tranformar el espacio universitario, como experiencia, en una instancia no solo educadora y creadora sino que además en una instancia de formación de ciudadanía. Así, la «ciudadanía universitaria» (Rivas, 2009) es una idea en construcción, porque es dinámica a los espacios, a las realidades y a las necesidades propias de los contextos universitarios, y porque se construye «comunidad universitaria» junto a todos sus actores: mundo académico, estudiantil, y funcionario.

Como se ha expuesto, estos conceptos resultan ser la esencia de aquello que hoy se plantea como democracia universitaria o democratización de los espacios universitarios, cuyo objetivo principal parece ser lograr la incorporación de los estudiantes en la elección de las autoridades y en los procesos de toma de decisiones, sin embargo, se advierte que este contenido es únicamente referencial pues la «democracia universitaria» como construcción teórica se encuentra aún en desarrollo.

Con todo, lo cierto es que en las universidades, la democracia no debe entenderse solamente como un aspecto político electoral, donde por las formas de la política tradicional se busque el poder dentro de las aulas o se agote en un ejercicio de mera elección popular. Corresponde un sentido decisional de mayor extensión y alcance que el electivo, ya que conlleva una responsabilidad mayor en virtud de perseguir fines superiores al representativo político, como enseñar, investigar y difundir conocimiento cultura (Olivera, Piña y Mercado, 2009).

\section{b) La participación estudiantil en la Universidad de Concepción}

i) Antecedentes de la UDEC

La UDEC es una universidad privada tradicional chilena, constituida como una corporación de derecho privado fundada el 14 de mayo de 1919. Es la tercera universidad más antigua de Chile, y una de las veinticinco universidades del Consejo de Rectores de las Universidades Chilenas. La UDEC ha centrado sus esfuerzos en tres ejes primordiales: enseñanza, investigación e innovación. Su impulsor principal fue el educador y abogado chileno Enrique Molina Garmendia, quien buscó crear la primera universidad laica de Chile. 
La casa central se ubica en la ciudad de Concepción, contando con otros dos campus en las ciudades de Chillán y Los Ángeles. Su campus central cuenta con tres vicerrectorías, 18 facultades, además del Centro de Biotecnología, Centro EULA y el Instituto GEA. La UDEC ha contado con diez rectores, cuatro de ellos fueron designados por la junta militar después de la eliminación de la elección democrática de autoridades universitarias en el año 1973.

\section{ii) La participación estudiantil en la UDEC}

En las universidades con las características que presenta la UDEC - una corporación de derecho privado reconocida por el Estado e integrante del Consejo de Rectores de las Universidades Chilenas (CRUCH) - esta forma de ejercer el poder de gobierno y su estructura, se encuentra determinada por sus propios estatutos, dando cuenta de una estructura organizativa y decisional de tipo vertical, cuyas decisiones se encuentran entregadas a la rectoría y consejo acádemico. La toma de decisiones en la universidad y el gobierno universitario corresponde casi exclusivamente al nivel directivo, contemplándose espacios de interacción con los estudiantes limitados al derecho de voz, y en ciertos casos, al derecho a voto, que de todas maneras no resulta ser determinante.

La interrogante que surge dice relación con los actores que determinan la forma en que se ejerce el gobierno universitario y la posible cabida en ello, del estamento estudiantil, en el entendido que el reclamo actual de los estudiantes se refiere precisamente a la posibilidad de influir de manera determinante en la toma de decisiones en los más diversos ámbitos del gobierno universitario, que se ha presentado bajo el concepto de «cogobierno universitario».

La UDEC tradicionalmente se ha constituido como una organización con una estructura claramente definida, encabezada por la rectoría y un consejo académico - formado esencialmente por los decanos de facultad - encargado de la toma de decisiones y presidido por el rector vigente en el cargo.

Ahora, en cuanto a la participación estudiantil y su caracterización, desde una perspectiva institucional y para determinar de manera precisa los espacios y formas de participación existentes en ella, debemos considerar que la participación estudiantil es un concepto multidimensional, que en el caso de la UDEC, comprende numerosos espacios de participación, caracterizados por ser espacios diversos, laicos y pluralistas. La UDEC, ha normado de manera estricta su funcionamiento a 
través de reglamentos universitarios, definiendo claramente los ámbitos de compentencia que corresponden a cada uno de los estamentos universitarios, además de determinar las formas de participación definidas para los estudiantes a través de reglamentos y políticas universitarias, las que en abundancia se han dirigido principalmente al fomento del deporte, la cultura, la interacción con la comunidad y la innovación, ciencia y tecnología como ámbitos destacados de la academia y la investigación. En el ámbito político, la situación es disímil, pues los espacios de participación no son abundantes. Sin embargo, se permite y asegura a los estudiantes el derecho a la libre asociación y organización a través de la federación de estudiantes a nivel macro, o a través de centros de alumnos a nivel micro. Igualmente, se han reglamentado aquellos espacios formales en que los estudiantes, y más bien, los representantes estudiantiles tienen participación, con derecho a voz, y en ocasiones con derecho a voto, en materias propias de la gestión académica y universitaria.

Las demandas estudiantiles en la UDEC, reclamadas fuertemente en el movimiento estudiantil del año 2012, han tenido como objeto principal, la democratización del gobierno universitario, solicitando la participación de los estudiantes en la elección democrática e inclusiva de las autoridades universitarias y la inserción del estamento estudiantil en la toma de decisiones.

Considerando que las demandas de participación estudiantil se han definido y circunscrito por los dirigentes estudiantiles de la UDEC al ámbito político, es que las líneas siguientes se centrarán en identificar los espacios de participación de los estudiantes en el ámbito político dentro de la estructura universitaria, desde una perspectiva transversal y el tipo de participación que en cada uno de ellos les corresponde.

Se distinguen espacios de elección y toma de decisiones en dos niveles, gobierno universitario y estudiantil. 


\section{A nivel universitario}

\section{Unipersonales:}

Rectoría: el rector es elegido democráticamente cada cuatro años por el cuerpo académico y representantes del directorio. Los académicos con derecho a voto son aquellos que cuentan con contrato indefinido y jornada académica de 22 horas o más. ${ }^{5}$

Decano de facultad: elegido democráticamente por el estamento académico. Designa al vicedecano, secretario académico y jefes de carrera.

Director de departamento: elegido democráticamente por los académicos pertenecientes a la planta del departamento con derecho a voto (contrato indefinido y jornada académica de 22 horas o más). ${ }^{6}$ En ninguna de estas instancias, los estudiantes tienen derecho a voz y voto.

Órganos colegiados:

Consejo ejecutivo universidad: órgano máximo de decisión, integrado por el rector, decanos de las facultades de los tres campus UDEC, vicerrectores y secretario general, además participan los representantes de la federación de estudiantes quienes sólo cuentan con derecho a voz.

Consejo directivo de facultad: integrado por el decano, quien lo presiden, exdecanos, directores de departamento, jefes de carrera y un representante estudiantil por cada carrera de la facultad con un máximo de tres. En este este consejo participa normalmente el presidente del centro de estudiantes o un integrante de su directiva. Consejo de carrera: ${ }^{7}$ existe en cada carrera de la universidad, constituye la instancia encargada de velar por el cumplimiento de los objetivos, evaluará los resultados y conocerá de cualquier problema interno de la carrera. Asimismo, diseñará las políticas de desarrollo de la carrera y de su proyección así como de la perti-

5 El estatuto de la Corporación de la Universidad de Concepción señala en su artículo 50 que «sólo podrán participar, en las elecciones de directivos de las facultades, los académicos que tengan nombramiento por media jornada o más en la universidad, además de la jerarquía y otros requisitos, en su caso».

6 Ibíd.

7 Artículo 8 Decreto Udec 2013-048. 
nencia de los planes de estudio. Lo integra el jefe de carrera, quien además lo preside, un mínimo de tres profesores de la facultad adscritos a la carrera, un mínimo de dos profesores de otras facultades que contribuyan significativamente al desarrollo de la carrera y por tres y hasta cuatro representantes estudiantiles de la carrera.

Comité de docencia y asuntos estudiantiles: ${ }^{8}$ existe en cada carrera de la universidad, constituye la primera instancia de resolución de las solicitudes presentadas por los alumnos y de consulta e información en caso de problemas o conflictos internos de las carreras. Lo integra el jefe de carrera, que lo preside, dos académicos elegidos por el consejo de carrera de entre sus integrantes y uno de los representantes estudiantiles que integra el consejo de carrera.

En los últimos tres casos, los representantes estudiantiles cuenta con derecho a voz y voto.

A nivel estudiantil:

Federación de Estudiantes de la Universidad de Concepción (FEC) $:{ }^{9}$ es una organización social y popular, que agrupa a todos los estudiantes y organizaciones de representación estudiantil de la UDEC, de forma democrática y federativa, promoviendo la organización de sus bases. La mesa directiva estará integrada por 5 miembros y los cargos serán los siguientes: presidente, vicepresidente, secretario general, secretario de finanzas y secretario de comunicaciones. Además cuenta con seis vocalías: cultura y deportes, trabajos comunitarios, derechos humanos, medio ambiente, solidaridad y conflictos, bienestar y académica. La mesa directiva y los vocales forman la mesa única de la federación, la cual se conformará además con los voceros, en caso de estado general de movilizaciones.

Consejo general de estudiantes (CGE): ${ }^{10}$ es el órgano dirigente, soberano y resolutivo de la federación entre congreso y congreso.

8 Artículo 9 Decreto UDEC 2008-116.

9 Estatuto Federación de Estudiantes Universidad de Concepción (FEC). Declaración de Principios, disponible en: www.udec.cl.

10 Artículo 20, Estatuto Federación de Estudiantes Universidad de Concepción. 
Son miembros del consejo general de estudiantes con derecho a voz y voto: los miembros de la mesa directiva de federación, un representante de cada centro de alumnos, un representante de cada carrera elegido en votación universal, un representante adicional, elegido por votación universal, en aquellas carreras con 350 alumnos o más. Los demás estudiantes sólo tendrán derecho a voz. Asamblea general de estudiantes: ${ }^{11}$ es la instancia máxima de la Federación de Estudiantes de la UDEC. Al ser convocada, determinará el rumbo de cualesquiera los temas que sean tratados y votados en ella, no pudiendo ninguna otra instancia de la federación contravenir las decisiones ahí tomadas. Son miembros de la asamblea general de estudiantes todos los estudiantes de la Universidad de Concepción, reconocidos por el su estatuto, los que tendrán derecho a voz y voto.

Centros de alumnos: cada carrera podrá conformar un centro de estudiantes, dictando las normas estututarias que regulen su conformación, funciones, atribuciones y renovación. En ella podrán participar todos los estudiantes de su carrera que gocen de la calidad de alumno regular de la UDEC y cumplan con los demás requisitos que señalen los respectivos reglamentos. En este contexto, en cada carrera los estudiantes podrán formular normas de organización política interna, considerando el establecimiento de funciones y competencias, la elección de delegados, Tricel, consejo de delegados, requisitos de conformación de grupos intermedios, etcétera.

Por último, cabe destacar que como resultado de las movilizaciones del año 2012, los dirigentes estudiantiles reconocen un avance en las demandas; luego de la negociación se logró el aumento de la participación de los estudiantes en los consejos de carrera, en el consejo de facultad aumentando de dos a cuatro representantes y consejo ejecutivo se logró la inclusión de los representantes de las federaciones de estudiantes de los campus Chillán y Los Ángeles.

11 Artículo 30, Estatuto Federación de Estudiantes Universidad de Concepción. 


\section{ANTECEDENTES ESPECÍFICOS DE LA INVESTIGACIÓN}

\section{a) Contexto del estudio y objetivos}

Durante el 2013, se ha llevado a cabo en la UDEC un estudio que se centra principalmente en caracterizar el concepto de «participación estudiantil» a nivel de educación universitaria, concebido por los estudiantes de pregrado de la UDEC. Para la realización de este estudio se ha contado con la participación de un equipo formado por estudiantes de la Carrera de Ciencias Políticas y Administrativas.

Se trata de un estudio descriptivo, cuyo diseño es no experimental, transeccional, orientado a estudiar el fenómeno de la participación estudiantil en el contexto de la Universidad de Concepción. La génesis de este estudio es a partir de la inquietud de demostrar que los estudiantes de la UDEC asocian principalmente el concepto de participación estudiantil orientándolo solo a los aspectos políticos y desconocen las formas de participación universitaria que entrega la UDEC.

La presente investigación pretende dar cumplimiento a los siguientes objetivos específicos: en primer lugar, identificar el grado de conocimiento que el estudiantado de la Universidad de Concepción posee sobre las formas de participación estudiantil en el contexto universitario. En segundo lugar, pretende identificar el grado de interés del estudiantado por la participación dentro de la Universidad de Concepción y finalmente determinar la percepción estudiantil respecto al nivel de influencia que tienen sus acciones en la toma de decisiones de la autoridad y los aspectos en que los estudiantes creen debiera tener lugar la participación estudiantil.

\section{b) Estudio: diseño, instrumentos y resultados}

En el desarrollo de la investigación se ha recurrido a diversas fuentes primarias de información, entre ellas: autoridades, académicos, estudiantes, bases de datos internas de estudiantes, documentación universitaria que regula los espacios de participación para los estudiantes en la Universidad, como reglamentos, estatutos, etcétera. Entre las estrategias utilizadas para la recolección de la información e instrumentos aplicados para la obtención se encuentran en primer lugar, la aplicación de un primer instrumento de tipo cuestionario para lograr captar los conceptos e ideas utilizados por los estudiantes en relación a la participación estudiantil, para posteriormente por medio de una red 
semántica se establecieran las palabras y oraciones utilizadas por los estudiantes, para que por medio de ellas, proceder a elaborar el instrumento de medición a aplicar. El instrumento final «La encuesta de participación estudiantil UDEC», fue elaborado por medio de preguntas de tipo test, cerradas del tipo dicotómica, de selección de alternativas y de preguntas con escalas de valoración de tipo likert. La encuesta fue aplicada mediante dos alternativas, la primera fue vía papel y la segunda vía electrónica, tanto por medio del correo electrónico como a través de las redes sociales. Ambas alternativas fueron vaciadas a la plataforma virtual para ser procesadas. Por otra parte, se aplicaron entrevistas personales por medio de cuestionarios con preguntas abiertas y semiestructuradas, a autoridades, entre ellos el vicerrector académico, el director de docencia, jefes de carrera, dirigentes estudiantiles y ex dirigentes estudiantiles.

Posteriormente, nos centramos en los resultados obtenidos mediante la aplicación de la encuesta en modalidad electrónica y en papel aplicada a los estudiantes de distintas carreras de la UDEC para conocer sus percepciones y en base a ellas establecer nuestra caracterización objetivo, apoyado de las entrevistas.

\section{c) La encuesta de participación estudiantil UDEC}

Los objetivos del instrumento aplicado son los siguientes: i) Identificar el grado de conocimiento que el estudiantado de la UDEC posee sobre las formas de participación estudiantil en el contexto universitario de la UDEC. ii) Identificar el grado de interés del estudiantado por la participación dentro de la UDEC. iii) Determinar cómo los estudiantes reconocen la importancia de influir mediante su participación en la toma de decisiones de las autoridades universitarias y los aspectos en que los estudiantes creen debiera tener lugar la participación estudiantil.

La encuesta contemplaba los siguientes aspectos en su estructura de seis partes con un total de 46 preguntas.

Primera parte: orientada a la identificación del encuestado, el objetivo de esta parte era obtener información en cuanto a género (sexo femenino o masculino), con ello podríamos inferir la tendencia en cuanto a género en cuanto al interés por responder el instrumento; carrera cuyo objeto era conocer el número de estudiantes del área de las ciencias sociales que daban respuesta al instrumento, para ello se clasificaron en carreras del área de las ciencias sociales y otras carreras; región de procedencia, para determinar si podía influir en la parti- 
cipación el hecho de que los estudiantes pertenecieran a la región o a otra región.

Segunda parte: orientada a obtener información respecto a la orientación de la conducta participativa de los estudiantes de la UDEC, para ello se establecieron cinco tipos de actividades asociadas a la participación estudiantil, el estudiante debía escoger aquella que de acuerdo a su criterio caracterizará más a la participación estudiantil, las actividades señaladas en la encuesta son: actividades de voluntariado, culturales, deportivas, políticas y académicas. Cada una de las actividades mencionadas tenía algún tipo de vinculación. Las actividades de voluntariado se vinculaban a la iglesia, a fundaciones privadas o al aparato gubernamental; las actividades culturales se vinculaban al teatro, música y artes; las actividades deportivas se vinculaban a la práctica de un deporte o a la observancia de una actividad deportiva u equipo deportivo; las actividades políticas se vinculaban al gobierno universitario, a la administración educacional, a las movilizaciones estudiantiles y a los liderazgos estudiantiles.

Tercea parte: preguntas 11 a la 18, orientadas a obtener información sobre el grado de información que los estudiantes tienen respecto de la participación estudiantil en la UDEC, valorar su importancia y el interés del estudiantado por participar en la UDEC.

Cuarta parte: preguntas 19 a 46, está formada por una serie de oraciones con respuesta de tipo Likert, cuya orientación indirectamente daría respuesta a la valoración de dichas afirmaciones con relación a si la participación estudiantil es reconocida por los estudiantes como gobernanza universitaria o cogobierno universitario.

\section{d) La población y la muestra}

La Universidad de Concepción cuenta con un universo de 23.130 estudiantes de pregrado matriculados para el período 2013. Considerando un margen de error de un 5\% y un nivel de confianza del 95\%, la muestra representativa correspondería a 378 individuos. Del universo, respondieron a la encuesta 402 estudiantes, durante un período de cinco días. De las 402 respuestas, 176 (44\%) corresponden a respuestas de estudiantes de sexo femenino y $226(56 \%)$ a respuestas de sexo masculino, de los cuales 233 respuestas $(58 \%)$ corresponden a carreras del área de las ciencias sociales y $169(42 \%)$ a carreras de otras áreas. De las 402 respuestas 276 (69\%) pertenecen a estudiantes provenientes de la Región del Biobío. 


\section{e) Presentación de resultados}

En términos generales, se observa que no hay diferencias significativas en función del género, sin embargo si se estima que hubo mayor interés por responder a esta encuesta por parte de los estudiantes que pertenecen a las carreras del área de las ciencias sociales.

i) Orientación de la conducta participativa

de los estudiantes de la Universidad de Concepción

Como se puede observar en el tabla 1, en la pregunta relacionada con la caracterización del concepto de participación estudiantil, se les presentó a los estudiantes una gama de cinco alternativas solicitándoles que marcaran solo aquella actividad que más caracteriza a la «participación estudiantil». La intención de esta pregunta era captar las tendencias de conducta participativa en los estudiantes encuestados. Del total de los encuestados, un $34 \%$ caracterizan el concepto como actividades políticas, un $27 \%$ como actividades académicas, un $18 \%$ como actividades deportivas, un $12 \%$ como actividades culturales, y un $10 \%$ como actividades de voluntariado.

TABLA 1: CONDUCTA PARTICIPATIVA

\begin{tabular}{|l|c|c|}
\hline ACTIVIDAD & FRECUENCIA & PORCENTAJE \\
\hline Políticas & 135 & $34 \%$ \\
\hline Académicas & 107 & $27 \%$ \\
\hline Deportivas & 72 & $18 \%$ \\
\hline Culturales & 47 & $12 \%$ \\
\hline Voluntariado & 41 & $10 \%$ \\
\hline
\end{tabular}

Actividades políticas: los estudiantes que escogieron esta alternativa, debían elegir además el ámbito al que se vinculaba la actividad política seleccionada, contando para ello con cuatro alternativas: La primera relacionada con el «gobierno universitario», entendiendo como tal a la inclusión de los estudiantes en actividades de participación establecidas formalmente dentro del contexto universitario, ya sea por reglamentos o estatutos, extendidos tanto por la autoridad universitaria de la UDEC, este tipo de actividades incluye la participación vinculante en la toma de decisiones por medio del comité de docencia de las carreras de pregrado, consejos de carrera de pregrado y los consejos de facultad. La segunda relacionada con la «administración educacional», la que incluye la participación en la elaboración de las políticas básicas 
de la educación, elaboración de programas curriculares, elaboración de programas educativos destinados a estudiantes, etcétera. La tercera vinculada a las «movilizaciones estudiantiles», entendiendo como tal la participación en actividades como protestas, marchas, paros y tomas de dependencias universitarias. La cuarta está vinculada a «liderazgos estudiantiles», entendiendo aquellas actividades de participación en centros de estudiantes, vocalías, consejos de delegados, etcétera.

El propósito de esta pregunta era determinar la tendencia de los estudiantes que habiéndose inclinado por una conducta participativa orientada a las actividades políticas, pudieran manifestar con cuál de esas actividades se sentían más identificados. Según lo indican los resultados, de total de los encuestados el $34 \%$ seleccionó la tendencia a las actividades políticas; de este alto porcentaje, el $43 \%$ se orientó a las actividades vinculadas a las movilizaciones estudiantiles, el $34 \%$ a las actividades vinculadas a liderazgos estudiantiles, el $13 \%$ seleccionó la opción vinculada a gobierno universitario y solamente el $10 \%$ optó por las actividades vinculadas a la administración educacional.

Actividades académicas: los estudiantes que seleccionaron esta alternativa, debían además cribar el ámbito al que mayormente se vinculaba. Se presentaban tres alternativas: La primera vinculada a las «responsabilidades de pregrado», orientadas principalmente a clases en aulas, actividades dentro y fuera del campus, trabajos en terreno, charlas, conferencias, etcétera. La segunda se vincula a «actividades de investigación», entendiendo como tales a la participación de los estudiantes en proyectos de investigación con apoyo de académicos u otros independientes para la participación en encuentros, congresos, etcétera. Y la tercera está vinculada «otras actividades académicas» como ayudantías, apoyo a actividades de extensión, asistencia técnica, miembros en grupos específicos orientados a la academia, etcétera.

El propósito de esta pregunta era determinar la tendencia de los estudiantes que habiéndose inclinado por una conducta participativa académica, pudieran manifestar con cuál de esas actividades se sentían más identificados. Según los resultados, del total de encuestados, el $27 \%$ seleccionó la tendencia a las actividades académicas; de este porcentaje, el $56 \%$ se orientó a las actividades vinculadas a las responsabilidades del pregrado, el $23 \%$ a las actividades vinculadas a investigación, el 13\% seleccionó la opción vinculada a gobierno universitario y el $21 \%$ optó por actividades vinculadas a otras actividades académicas.

Actividades deportivas: los estudiantes que seleccionaron esta alternativa, se le presentaron tres opciones: La primera vinculada a la 
«práctica de un deporte», la segunda se asocia a «observar un deporte o seguir a un equipo deportivo» y la tercera a «otras actividades con orientación deportiva», como por ejemplo ser dirigente deportivo o participar en organizaciones deportivas.

La meta de esta pregunta era poder determinar la tendencia de los estudiantes que habiéndose inclinado por una conducta participativa deportiva, pudieran manifestar con cuál de esas actividades se sentían más identificados. Según los resultados, del total de encuestados, $18 \%$ seleccionó la tendencia a las actividades deportivas, de este porcentaje, el $88 \%$ se orientó a las actividades vinculadas a la práctica de un deporte, y sólo un $12 \%$ cribó las actividades vinculadas a observar un deporte o seguir a un equipo deportivo. Ninguno de los estudiantes encuestados optó por seleccionar otras actividades deportivas.

Actividades culturales: los estudiantes que se inclinaban por esta alternativa, debían escoger el ámbito cultural de la actividad seleccionada, contando para ello con cuatro ámbitos a saber: La primera vinculada a la «música», la segunda se vincula a «las artes (pintura, plástica, gráficas, etcétera)», la tercera está vinculada «al teatro» y una cuarta opción está orientada a «otras actividades culturales», como por ejemplo la fotografía, bibliotecas, hemerotecas, pinacotecas, etcétera.

La finalidad de esta interrogante, era poder determinar la tendencia de los estudiantes que habiéndose inclinado por una conducta participativa cultural, pudieran manifestar con cuál de esas actividades se sentían más identificados. Según los resultados, del total de encuestados, el $12 \%$ seleccionó la opción tendiente a las actividades culturales; de este porcentaje, el $70 \%$ se orientó a las actividades vinculadas a la música, el $15 \%$ a las actividades vinculadas a las artes, el $11 \%$ al teatro y sólo un $4 \%$ se identifican con otras actividades culturales.

Actividades de voluntariado: los estudiantes que optaron por esta alternativa, debían escoger entre aquellas actividades en que se desarrollara el voluntariado, presentándose para ello cuatro posibilidades: La primera vinculada a la «iglesia»; es decir, actividades de voluntariado de ayuda al prójimo mediante grupos organizados con estudiantes de la UDEC a través de alguna organización eclesiástica; la segunda se vincula a las «fundaciones privadas»; la tercera al aparato gubernamental» (todas aquellas actividades que se realizan a través de la municipalidad, intendencias o a través de la ejecución de alguna política pública transversal) y una cuarta opción está orientada a «otras actividades de voluntariado». 
Se buscaba determinar la tendencia de los estudiantes que habiéndose inclinado por una conducta participativa al voluntariado, pudieran manifestar con cuál de esas actividades se sentían más identificados. De acuerdo a los resultados observados, del total de los encuestados el $10 \%$ optó por la tendencia a las actividades de voluntariado; de este porcentaje el $34 \%$ se orientó a las actividades vinculadas al voluntariado por medio de alguna organización eclesiástica, el 32\% a las actividades asociadas a fundaciones privadas, el $10 \%$ al aparato gubernamental y el $24 \%$ de ellos se identifican con otras actividades de voluntariado.

ii) Información sobre el conocimiento que tienen los estudiantes de la Universidad de Concepción con respecto a la participación estudiantil en la UDEC

Adicionalmente se pretendía obtener información respecto al conocimiento que tienen los estudiantes de la participación estudiantil en la UDEC. En este orden de cosas, al preguntar a los estudiantes si conocían las formas de participación que ofrece la UDEC, del total de encuestados el $76 \%$ tiene una respuesta afirmativa en esta materia; en contrapartida, un porcentaje que no supera el $25 \%$ indican NO conocer las formas de participación.

TABLA 2: CONOCIMIENTO DE FORMAS DE PARTICIPACIÓN EN LA UDEC

\begin{tabular}{|l|c|c|}
\hline CONOCIMIENTO & FRECUENCIA & PORCENTAJE \\
\hline Sí & 307 & $76 \%$ \\
\hline No & 95 & $24 \%$ \\
\hline
\end{tabular}

Además se le solicita a los encuestados que califiquen el nivel de importancia de las formas de participación política reguladas en la UDEC, en nivel muy importante, importante, medianamente importante y menos importante. Como puede desprenderse de la tabla 3, la figura más valorada de participación es la asamblea, ya que del total de encuestados el $46 \%$ las pondera como «muy importantes» y para el $31 \%$ de ellos es «importante». Lo anterior, permite deducir que los estudiantes valoran mayormente aquellas formas de participación en la que tienen un mayor espacio o una participación masiva, donde pueden hacer valer los derechos de voz y voto. 
TABLA 3: NIVEL DE IMPORTANCIA SEGÚN LOS ESTUDIANTES ENCUESTADOS

\begin{tabular}{|l|c|c|c|c|c|}
\hline $\begin{array}{l}\text { FORMA DE } \\
\text { PARTICIPAR }\end{array}$ & $\begin{array}{c}\text { MUY } \\
\text { IMPORTANTE }\end{array}$ & IMPORTANTE & $\begin{array}{c}\text { MEDIANAMENTE } \\
\text { IMPORTANTE }\end{array}$ & $\begin{array}{c}\text { MENOS } \\
\text { IMPORTANTE }\end{array}$ & $\begin{array}{c}\text { TOTAL } \\
\text { ENCUESTAS }\end{array}$ \\
\hline $\begin{array}{l}\text { Comité de } \\
\text { docencia }\end{array}$ & 130 & 190 & 51 & 31 & 402 \\
\hline $\begin{array}{l}\text { Consejo de } \\
\text { carrera }\end{array}$ & 137 & 178 & 70 & 17 & 402 \\
\hline $\begin{array}{l}\text { Consejo de } \\
\text { facultad }\end{array}$ & 126 & 154 & 91 & 31 & 402 \\
\hline $\begin{array}{l}\text { Consejo de } \\
\text { delegados }\end{array}$ & 90 & 147 & 124 & 41 & 402 \\
\hline $\begin{array}{l}\text { Centro de } \\
\text { alumnos }\end{array}$ & 167 & 147 & 68 & 20 & 402 \\
\hline Asambleas & 183 & 125 & 66 & 28 & 402 \\
\hline
\end{tabular}

También interesaba conocer la opinión respecto de si los medios de difusión en la UDEC son conocidos por todos los estudiantes. Se determinó que a lo menos el 33\% de los encuestados reconoce que los medios de difusión son conocidos por todos los estudiantes. Sin embargo, llama la atención que el 35\% de ellos señala no estar de acuerdo con esta afirmación y un porcentaje casi similar (32\%) se mantiene neutro ante esta afirmación indicando «ni de acuerdo, ni en desacuerdo».

iii) Importancia de la participación estudiantil e interés por participar que tienen los estudiantes de la Universidad de Concepción

Como se puede observar en la tabla 4, prácticamente el $42 \%$ considera «sumamente importante» la participación estudiantil, y un poco más del 33\% «indispensable»; en contrapartida, un porcentaje que no supera el 5\% lo evalúa como «poco importante».

TABLA 4: NIVEL DE IMPORTANCIA DE LA PARTICIPACIÓN EN LA UNIVERSIDAD, SEGÚN LOS ESTUDIANTES ENCUESTADOS

\begin{tabular}{|c|c|c|c|c|}
\hline INDISPENSABLE & $\begin{array}{c}\text { SUMAMENTE } \\
\text { IMPORTANTE }\end{array}$ & $\begin{array}{c}\text { MEDIANAMENTE } \\
\text { IMPORTANTE }\end{array}$ & $\begin{array}{c}\text { POCO } \\
\text { IMPORTANTE }\end{array}$ & $\begin{array}{c}\text { No SE TOMA } \\
\text { EN CUENTA }\end{array}$ \\
\hline 134 & 167 & 75 & 20 & 6 \\
\hline
\end{tabular}

Por otra parte, según lo muestra la información del tabla 5, el 76\% de los encuestados indica tener participación estudiantil en la UDEC en algunas de sus formas y tan sólo el 24\%, dice no participar. 
TABLA 5: PARTICIPACIÓN EN LA UDEC

\begin{tabular}{|l|c|c|}
\hline PARTICIPA & FRECUENCIA & PORCENTAJE \\
\hline Sí & 299 & $74 \%$ \\
\hline No & 103 & $26 \%$ \\
\hline
\end{tabular}

\section{CONCLUSIONES}

El estudiante UDEC, reconoce principalmente como la más importante figura de participación estudiantil a la participación orientada a actividades políticas, entendiéndose como tales para objeto de este estudio a todas aquellas que hemos llamado gobierno universitario, que incluye participación vinculante en la toma de decisiones universitarias por medio de los comités de docencia de pregrado, consejo de carrera y los consejos de facultad. Incluso, la mayoría de los estudiantes cuya conducta participativa se inclina a actividades políticas, se identifica principalmente con actividades relacionadas con las movilizaciones estudiantiles las que se encuentran dirigidas, principalmente al logro de demandas de una mayor vinculación y espacios de decisión para los estudiantes.

El estudiante UDEC, asocia claramente la participación estudiantil a la construcción de la democracia universitaria, donde esta participación debe ser principalmente vinculante en la toma de decisiones de la autoridad universitaria. La gran mayoría de los estudiantes (68\%) piensa que las autoridades universitarias deberían ser escogidas mediante participación democrática a través de un mecanismo que permita su inclusión en este proceso.

El estudiante UDEC, valora y reconoce el espacio de participación que les otorga la figura de la «asamblea», ya que esta representa el interés de los estudiantes, les permite escuchar a los demás, conocer la opinión de sus compañeros, opinar, votar y también llegar a consensos respecto de los aspectos universitarios que son de su interés.

El estudiante UDEC, no asocia el aumento de poder en el estudiante por medio de la participación como un hecho que vaya a sugerir aumento de conflictos, sino que valoran enormemente la posibilidad de diálogo concreto, de hecho más del 50\% de los estudiantes asocia la participación con influir en las decisiones universitarias y no se excluye de participar, aunque exista la posibilidad de que sus opiniones no sean tomadas en cuenta. 
El estudiante UDEC, considera que las «tomas y marchas» NO son más efectivas que el diálogo, buscando siempre espacios democráticos. Los estudiantes UDEC, consideran que mediante la participación estudiantil ellos podrán tener voz dentro de los cambios estructurales y en la construcción de una nueva sociedad. Por otra parte, es destacable mencionar que a los estudiantes les interesa participar de la creación curricular de sus mallas y programas curriculares, pues son los principales afectados con este tipo de procesos.

De lo anterior, podemos concluir que principalmente los estudiantes UDEC asocian el concepto de participación estudiantil orientándolo a actividades por una parte vinculadas a la academia (pregrado) y por otra parte vinculadas a la política. En general, tienen claras las formas de participación que entrega la UDEC y reconocen la importancia de influir mediante su participación en la toma de decisiones de las autoridades universitarias. Sin embargo, respetan a la autoridad y esperan contar con mayores espacios de participación política dentro de los medios establecidos por la reglamentación Universitaria a través de opinión con propuestas fundadas en actitudes recogidas democráticamente dentro del estudiantado (asambleas), las que esperan sean vinculantes en la toma de decisiones de la autoridad. Lo que nos hace concluir que el concepto de participación estudiantil - para los estudiantes UDEC - se encuentra asociado principalmente a lo que conocemos como cogobierno universitario.

Las expectativas del estamento estudiantil en cuanto a lograr un cogobierno universitario encuentra, como hemos señalado anteriormente, una serie de trabas no sólo internas, en tanto que para generar estos espacios de mayor participación y toma de decisiones se requiere de la derogación y dictación de nuevos reglamentos corporativos y universitarios al interior de la UDEC, sino que también externas, pues la falta de una política pública para la educación superior en aspectos esenciales como lo son el fomento de la participación, la democracia, la vinculación de los estudiantes universitarios con la sociedad y el rol esencialmente social que tienen estas instituciones de aprendizaje y formación en la construcción de profesionales y ciudadanos activos, comprometidos, conscientes de su rol en la sociedad y con cultura política para asegurar el futuro de una sociedad democrática, no ha sido abordada por las autoridades políticas de manera seria.

La falta de espacios vinculantes en que los estudiantes puedan participar y percibir la importancia de su actividad a nivel universitario, provoca un creciente desinterés de los estudiantes en participar en 
el ámbito más político de su quehacer universitario, privándolos además de desarrollar una serie de habilidades, muy valoradas en el campo laboral y que de otra forma son muy difíciles de alcanzar como el liderazgo, la expresión oral, el trabajo mancomunado, la capacidad de negociación y llegar a consenso, etcétera, por ello es de vital importancia la construcción de una democracia universitaria inclusiva y participativa que recoja la diversidad de los estudiantes que forman parte de la comunidad y que contribuya positivamente a su formación profesional y cívica.

CONCEPCIÓN (CHILE), JULIO 2014

RECIBIDO: JULIO 2014 ACEPTADO: ENERO 2015 


\section{REFERENCIAS BIBLIOGRÁFICAS}

Andolina, Molly W., Krista Jenking, Cliff Zukin y Scott Keeter (2003): «Habits from Home, Lessons from School: Influences on Youth Civic Engagement». PS: Political Science \& Politics 36.

Astin, AleXANDER W., Linda J. SAX y JuAn AValos (1999): «The LongTerm Effects Of Volunteerism During the Undergraduate Years». The review of higher education 21(2).

Bobbio Norbeto (2000): El futuro de la democracia. México: Fondo de Cultura Económica.

BRUNNER, JOSÉ JOAQUín (1990): Educación superior en América Latina: Cambios y desafíos. Santiago: Fondo de Cultura Económica.

- (2011): «Gobernanza universitaria: tipología, dinámicas y tendencias». Revista de Educación.

CONFECH (2012): «Historia de la CONFECH y el movimiento universitario chileno en los últimos 30 años».

DAHL, ROBERT (1999): La democracia. Una guía para los ciudadanos. Buenos Aires: Editorial Taurus.

DECLARACIÓN DE BERGEN (2005): «El espacio europeo de educación superior: alcanzando las metas». Comunicado de la conferencia de ministros europeos responsables de educación superior. Bergen, 19-20 de mayo.

DECLARACIÓN DE BERLÍN (2003): «Educación superior europea». Comunicado de la conferencia de ministros responsables de la educación superior. Berlín, 19 de septiembre.

DEClARACIÓN DE PRAGA (2001): «Hacia el área de la educación superior europea». Declaración del encuentro de los ministros europeos en funciones de la educación superior en Praga, 19 de mayo.

DiodATO, JoRge Luis y VALERIA MARTíNEZ DEL SEL (2006): «Autonomía y el cogobierno universitario: repensando su sentido reformista en los estatutos». Buenos Aires: Universidad de Buenos Aires.

KRAUSKOPF, DiNA (1998): «Dimensiones críticas en la participación social de las juventudes». En: Participación y desarrollo social en la adolescencia. San José: Fondo de Población de Naciones Unidas.

MARTín, I. (2007): «La participación política de los estudiantes universitarios dentro y fuera de la universidad». Panorama Social $\mathrm{N}^{\circ} 6$.

MERHI AUAR, RICHARD (2011): «Las claves de la participación estudiantil en las universidades españolas». Girona: Universidad Jaume I.

RIVAS PALMA, GlORIA (2009): «Clima emocional en el aula un nuevo concepto como indicador de desempeño académico». Concepción: Universidad del Biobío.

MinISTERIO DE EDUCACIÓN (1990): «Ley Nº18.962 Orgánica Constitucional de Enseñanza (LOCE)».

- (2005): «DFL N ${ }^{\circ} 1$ Fija texto refundido, coordinado y sistematizado de la Ley $N^{\circ} 18.962$, Orgánica Constitucional de Enseñanza». 
— (2009): «Ley N²0.370 establece la Ley General de Educación». (2010): «DFL No2 Fija texto refundido, coordinado y sistematizado de la Ley $\mathrm{N}^{\circ} 20.370$ con las normas no derogadas del Decreto con Fuerza de Ley $\mathrm{N}^{\circ} 1$ de 2005 ».

NAVARRO, G. (2012): «Moralidad y responsabilidad social: bases para su desarrollo y educación». Concepción: Universidad de Concepción.

OLIVERA, PIÑA y MERCADO (2009): «La universidad pública: autonomía y democracia». Convergencia, Revista de Ciencias Sociales No51. México: Universidad Autónoma del Estado de México.

PROGRAMA DE NACIONES UNIDAS PARA EL DESARROLlO (2002): «El enfoque de gobernabilidad democrática del PNUD». Santiago: PNUD.

TOMASSINI, LUCIANO (1993): «Estado, gobernabilidad y desarrollo». Banco Interamericano de Desarrollo.

VICERRECTORÍA UNIVERSIDAD DEL PAÍS VASCO (2012): «Primer plan de participación del alumnado de la UPV/EHU». 